\title{
Adolfo Vera - Recherche autour de la question de la disparition politique -2008
}

\section{Adolfo Vera}

\section{(2) OpenEdition}

\section{Journals}

Édition électronique

URL : http://journals.openedition.org/appareil/671

DOI : 10.4000/appareil.671

ISSN : 2101-0714

\section{Éditeur}

MSH Paris Nord

\section{Référence électronique}

Adolfo Vera, «Adolfo Vera - Recherche autour de la question de la disparition politique - 2008 », Appareil [En ligne], Articles, mis en ligne le 21 novembre 2008, consulté le 22 septembre 2020. URL http://journals.openedition.org/appareil/671 ; DOI : https://doi.org/10.4000/appareil.671

Ce document a été généré automatiquement le 22 septembre 2020.

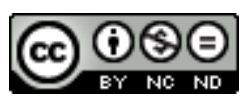

Appareil est mis à disposition selon les termes de la Licence Creative Commons Attribution - Pas d'Utilisation Commerciale - Pas de Modification 4.0 International. 


\title{
Adolfo Vera - Recherche autour de la question de la disparition politique -2008
}

\author{
Adolfo Vera
}

\section{Introduction}

Notre projet de recherche s'inscrit dans le contexte d'une discussion qui a été développée en l'Amérique Latine et en Europe depuis quelques décennies, et qui a pour enjeu fondamental de mettre en rapport des catégories esthétiques (propres à ce qu'on appelle plus largement Philosophie de l'art) avec des catégories de la Philosophie politique, pour comprendre, d'un point de vue esthético-politique, des phénomènes qui s'insèrent dans le contexte de situations vécues par les populations sous les régimes dits «totalitaires». Dans ce contexte très large, notre intérêt spécifique porte sur le problème de la "disparition ", phénomène qui a été considéré selon la théorie des totalitarismes comme l'un des plus pratiqués par les régimes totalitaires, et qui consiste - pour le dire dans un mot - en la stratégie policière qui efface toutes les traces d'une victime d'un crime politique, pour en empêcher toute possibilité d'une reconnaissance postérieure du crime. Cette stratégie, on le sait, a été pratiquée dans la première fois par les nazis dans leur programme d'extermination des juifs d'Europe, programme connu comme la «Solution Finale». Notre recherche s'est concentrée sur le phénomène de la « disparition » propres aux dictatures latino-américaines des années 70 et 80, notamment les dictatures argentine (1976-1983) et chilienne (1973-1990). Néanmoins, quoique le contexte historique et l'analyse qui en a été faite soient fondamentaux, notre accent théorique est avant tout esthétique (il s'agit pour nous de rapprocher les problématiques esthétiques de celles de la politique). Donc notre recherche va se concentrer, d'abord, sur une détermination philosophique assez précise de la catégorie de "disparition " - en la mettant en rapport avec d'autres catégories centrales de la philosophie contemporaine comme celles d'événement, mémoire, témoignage, communauté -, ensuite sur une analyse d'œuvres et d'artistes 
latino-américains qui ont travaillé sur la "disparition ». Parallèlement, notre objectif est - à partir de ces discussions - de définir un type d'image artistique spécifique que nous appelons "l'image politique». Les catégories philosophiques de mémoire et d'institution serviront pour (toujours guidées par celle de la disparition) complexifier une problématique qui est, nous semble-t-il, de la plus grande importance pour la discussion actuelle à propos des questions de la politique et de l'art en Amérique latine.

Cette recherche de formation doctorale dans notre carrière professionnelle, en considérant nos recherches antérieures (surtout celle qui nous a conduit à l'obtention du Master à l'Université du Chili) est un moment crucial, non pas seulement par l'envergure du travail de recherche à réaliser, mais surtout parce que nous réalisons notre travail avec l'un des théoriciens qui a le plus contribué à une définition philosophique de la "disparition", dans ses ouvrages mais aussi, dans plusieurs colloques, ouvrages collectifs, thèses dirigées, etc. qu'il a mené. Enfin, nous pouvons ajouter que cette recherche a pour but - et importance - le fait de contribuer à la discussion de ces problèmes esthético-politiques dans le contexte de la philosophie chilienne, et plus largement latino-américaine, sujet qui, paradoxalement, n'a pas encore été beaucoup travaillé sur place à partir de la question de la disparition. Cela sera l'un de nos objectifs avant de retourner dans notre pays d'origine une fois terminée notre plan de formation doctorale.

\section{Résumé du travail réalisé}

$\mathrm{Au}$ cours de cette première année de formation doctorale nous avons effectué un travail d'élaboration philosophique précise des catégories de la «disparition » et de l'«image politique». Dans un premier temps (nous en reparlerons aux points "conclusions» et "chronogramme d'activités»), nous avons tenté d'effectuer une analyse de ce que nous appelons "l'image politique», à partir d'une certaine «philosophie du désir » exprimée par les philosophes Deleuze et Lyotard, et qui définit l'image comme "fantasme" (à partir des termes psychanalytiques Freudiens et Lacaniens). Ces analyses ont été le motif d'un exposé dans le Séminaire des doctorants de l'équipe "Arts, appareils, diffusion" de la MSH Paris-Nord et du Laboratoire Logiques contemporaines de la philosophie de l'Université Paris 8, séminaire dirigé par le Professeur Jean-Louis Déotte avec la collaboration du Professeur Alain Brossat et de Véronique Fabbri. L'exposé a été présenté en février 2008. Nous avons reçu de nombreuses critiques (notamment de J-L Déotte et V. Fabbri) surtout pour n'avoir pas centré l'analyse sur le sujet de la disparition, une "philosophie de l'image désirante " comme fantasme n'ayant rien à voir avec le sujet spécifique de la disparition.

Depuis nous avons centré nos analyses sur le sujet spécifique de la disparition et de l'image politique comme image des «fantômes " (nous sommes donc passé de l'image comme «fantasme» à l'image comme «fantôme»; voir plus bas le point «Discussion»), à partir de la lecture de Gilbert Simondon, Walter Benjamin, J-F. Lyotard, Jacques Derrida, Jacques Rancière, Georges Didi-Huberman, J-L. Déotte parmi d'autres, et de l'analyse d'œuvres cinématographiques, notamment Nuit et Brouillard et Hiroshima mon amour d'Alain Resnais, Histoire(s) du cinéma de Jean-Luc Godard, Shoah de Claude Lanzmann. 
D'autre part, nous avons assisté à plusieurs colloques, journées d'études et séminaires de l'École Doctorale de Paris 8 et de la Maison de Sciences de l'Homme Paris-Nord.

\section{Objectifs du Projet}

1. Détermination précise des catégories esthético-politiques concernant le sujet de la «disparition». Il s'agit de développer théoriquement des catégories comme celles d'événement, de trace, de mémoire, de surface d'inscription, de ruine, de désastre, de catastrophe, pour en déterminer philosophiquement, et avec précision et rigueur, d'un point de vue esthetico-politique, la question de la « disparition ».

2. Contribuer à l'ouverture d'un axe de recherche au Chili, versant sur le sujet de la disparition comme définie dans ce rapport. C'est l'objectif essentiel du projet de formation, que nous pourrons le mettre au point lors du retour à notre Institution d'origine (Université de Valparaíso, Chili).

3. À partir de ce qui a été mentionné en a) et b) : commencer une carrière de chercheur dans notre pays d'origine, en considérant nos recherches antérieures mais surtout à partir des problématiques esthético-politiques que nous étudierons dans ce projet de formation.

\section{Méthodologie}

Jusqu'ici nous avons utilisé une méthodologie propre aux recherches philosophiques, c'est-à-dire, notamment à partir de l'étude des textes (la bibliographie) soit recommandés par notre Directeur de Recherche, soit étudiés dans les séminaires suivis; mais aussi à partir de l'élaboration des schèmes thématiques qui doivent guider la rédaction des textes de notre recherche (nous avons d'ores et déjà un plan de travail pour rédiger d'ici à la fin de l'année une centaine de pages, dont une quarantaine est déjà rédigée). Ce travail a toujours été dirigé sur la base d'hypothèses mises à l'épreuve avec notre tuteur.

\section{Résultats et discussion}

Il faudra toujours penser la disparition en mettant en crise la pensée. La disparition est ce qui, dans notre temporalité, a mis en question la temporalité elle-même, si l'on considère cette temporalité du point de vue de la "philosophie de l'histoire » moderne (des linéarités téléologiques des événements, continuité des processus, ou, au moins, superposition, couches de savoir-pouvoir, en constituant « notre » expérience des êtres historiques : généalogie, archéologie du savoir) c'est là où la disparition agit comme bouleversement radical de "notre " expérience, comme l'événement qui pourrait arriver jusqu'à annuler tout événement - l'(anti)événement, donc du désastre (Blanchot) -. On pourrait dire, comme l'a fait Jean-Louis Déotte, que ce qui finalement arriverait à "interrompre l'histoire» ne serait pas le «Messie», ni le communisme, mais, sous le signe de la Catastrophe, le fait de la stratégie policière à partir de laquelle, dès les nazis jusqu'aux militaires argentins et chiliens, en passant par les militaires français en Alger, une femme, un homme, un enfant, ont pu être pris et disparaître, sans après, (mais après quoi? que s'est-il passé là? Où est mort l'événement?) ; ils ont disparu, sans laisser de traces, pour ne revenir que comme des fantômes qui gênent notre "paix sociale", pour en montrer, en tout cas, l'impossibilité même de la 
communauté. Il s'agira donc d'amener la pensée politique jusqu'à une pensée des fantômes, pour en constituer une "philosophie politique du fantôme ", en tant que motif central de toute pensée de la politique - de ce qui pourra être, aujourd'hui, une communauté - « après la catastrophe ».

Nous commencerons par établir ce que nous appellerons «l'image politique». Une image politique (toute image n'étant pas politique) sera, selon nous, une image qui rend compte du processus politique d'anéantissement du sens, que nous appellerons en suivant J.-L. Déotte et d'autres, la catastrophe, c'est-à-dire la crise radicale du sens après l'horreur vécue par des milliers de victimes des totalitarismes depuis Auschwitz jusqu'à nos jours. Nous considérons ainsi, qu'après Auschwitz, toute idée de communauté politique, ne peut être pensée, analysée, postulée, etc., qu'au cœur de cette pensée-là, le désastre du sens, noyau même de la politique en tant qu'exercice (toujours paradoxal et fondé sur la mésentente selon Rancière) de l'être ensemble de la communauté, fait de l'effondrement du sens. Nous serons alors autorisés à dire qu'une image qui n'est pas née de cette problématique-là, ne sera pas une « image politique ». Ce seront des images publicitaires, des images scientifiques, des images mentales, etc., en aucun cas des images politiques. Il se trouve que ce type d'image, né d'une détermination assez précise, est une définition du fait politique du "nous " (nous utiliserons ce "nous» en suivant Lyotard, entre guillemets) qui a été développé, travaillé et créé spécifiquement dans l'art contemporain. Nous savons que l'effet le plus net de la fameuse sentence d'Adorno à propos de l'impossibilité de l'art après Auschwitz, a été justement que l'art postérieur à cette période n'a fait que s'exercer sur ce que nous appellerons dans cette recherche, en suivant Blanchot le désastre. C'est pour cela que selon cette hypothèse, la philosophie politique du fantôme ne pourra être en même temps qu'une philosophie de l'art « après l'époque de la disparition » (Déotte).

Ce n'est pas par hasard, alors, que la «forme artistique " la plus importante de la période postérieure à Auschwitz est la photographie. La photographie, art de la trace, a été développée de la façon la plus intense à une époque où le problème politique fondamental était précisément celui de «l'absence de traces». On se trouve, après Auschwitz, en présence d'un mythe, raconté par un auteur du siècle premier de notre ère, Pline l'ancien, qui apparaît surchargé de connotations politiques: l'histoire de Dibutade. Pour les familiers des disparus - par exemple pour ceux des disparus argentins ou chiliens - il s'agissait de, à partir de l'appareil photographique et ses caractéristiques essentielles, c'est-à-dire, parmi d'autres, la temporalité anachronique, la reproductibilité technique, la conformation de ses images en tant qu'Index qui rendaient possible l'expérience du ça a été (Barthes), de montrer et de démontrer à la communauté déjà brisée, à chaque fois, par l'exécution d'un nouveau rite, que ces enfants, ces maris, ces épouses, ces parents avaient eu un visage, un corps, un nom, en un mot - et pour utiliser le concept sans encore le définir - une trace. Il s'agissait donc de revenir, à chaque fois, au rituel de Dibutade, de tracer sur un support - le mur, le papier - l'inscription d'un corps, pour le remémorer, pour savoir qu'il avait existé, qu'il avait vécu. Ce rituel a été transformé, pour les familiers des disparus, en rituel presque épique - et on dira "presque» puisque, dans une communauté brisée et sans destination claire du fait de l'entrecroisement entre la terreur d'état et le capitalisme sauvage, l'épique ne peut plus avoir lieu - pour montrer et démontrer, face à la puissance de la disparition, que ces personnes-là avaient existé. 
Il faudra, donc, déterminer le moment politique - au sens où nous avons défini l'image politique - des théories sur la photographie qui ont mis l'accent sur le caractère indiciel de l'image photographique - nous pensons là surtout aux travaux de Rosalind Kraus, Philippe Dubois et Roland Barthes.

La question proprement philosophique va, plus loin : quel est le rapport entre trace, événement, inscription (et donc surface d'inscription)? Peut-on considérer la disparition comme un événement? Ne serait-elle pas plutôt (comme on l'a déjà dit) un anti-événement, l'événement qui annule toute possibilité d'événement, puisqu'il annule la possibilité de l'inscription d'une trace sur une surface, en annulant la trace et la surface (le corps et l'empreinte du corps) ? L'effort des familiers, la pratique rituelle systématique (les danses, les chansons, les déguisements), ne sont-ils pas, à proprement parler, des gestes et des actions politiques pour faire apparaître l'événement - de la disparition et de la vie antérieure à la disparition -, pour l'inscrire dans de nouvelles surfaces d'inscription (les monuments, les rites dans les rues, les institutions Fundación Madres de la Plaza de Mayo, Asociación de Familiares de Detenidos Desaparecidos parr exemple) ? La question est : comment faire apparaître l'événement qui a disparu (l'événement d'une vie) ou qui apparaît seulement de façon négative (l'anti-événement de la disparition)? Nous allons essayer d'y répondre à travers l'analyse de ce qui a été l'expérience de l'art et la littérature latino-américaine qui, à partir des années 70, a travaillé systématiquement sur ces problématiques.

La disparition, parallèlement, peut être comprise comme interruption des processus sociaux d'individuation - comme crise radicale de ce qui permet la constitution d'une " communauté ». Nous travaillerons fondamentalement avec la notion d'individuation psychosociale chez Gilbert Simondon. Cette théorie, nous semble-t-il, va nous permettre de comprendre comment ce qu'on peut considérer comme le processus nucléaire de constitution d'une communauté - et d'une individualité donc, en sachant qu'une individualité ne peut pas se constituer sans rapport à la communauté, et vice versa - va être écrasé du fait de la disparition. Toute communauté, selon Simondon, se constitue à partir de processus de "métastabilité » sociale, c'est-à-dire de crises continuelles de l'identification des sujets sociaux aux institutions, aux lois et normes qui engendrent la vie en commun. La métastabilité y indiquerait le fait que la sociabilité est produite par la crise et non pas par la stabilité. Cependant, la disparition ruine cette métastabilité, puisqu'elle ruine la possibilité même de la reconnaissance d'un individu face à un autre comme membre d'une communauté, puisque à «l'époque de la disparition" nos sociétés doivent vivre avec des fantômes, c'est-à-dire, selon la terminologie de Nicolas Abraham et Maria Torok, avec des morts qui ne sont pas «bien morts » et qui reviennent toujours vers nous, des morts « intolérables ». Simondon luimême insistait sur le fait que face aux morts le processus d'individuation ne s'arrête pas, puisque les vivants continuent, non seulement avec les rites mortuaires, mais aussi à partir de tout ce qu'on peut considérer comme le processus social du souvenir. Or, à partir de Simondon on pourra dire qu'un des faits anthropologiques les plus importants - celui du rapport d'individuation entre la communauté des vivants et la « communauté » des morts - en sera interrompu du fait de la disparition. Cela va mettre en crise le processus même d'individuation psychosociale. La disparition laisse donc le sujet au moment du pré-individuel, qui selon Simondon est le moment de l'affectivité pure. Or, dans les sociétés qui se développent sous des régimes qui pratiquent systématiquement la stratégie de la disparition, comme stratégie politique de la terreur, (et dans une certaine mesure dans les sociétés postérieures - même 
démocratiques - y reviennent sans cesse des fantômes - ceci ne s'arrêtera jamais), va s'imposer ce que J-L Déotte appelle la "désindividuation sociale", c'est-à-dire l'expérience d'une affectivité psychosociale ancrée dans l'angoisse et la terreur.

Pointe une autre des questions fondamentales de notre recherche: qu'est-ce qu'une communauté ? Après le développement systématique de la stratégie de la disparition par les dictatures latino-américaines des trois dernières décennies, toute pensée intéressée par la problématique du politique devra y faire retour. Le politique apparaît toujours comme une certaine lutte pour les espaces même antérieurs au discours, les espaces propres à l'installation de la sensibilité, donc comme une crise toujours renouvelée par l'exercice politique, puisque la crise elle-même, ce que Rancière appelle la mésentente, y constitue l'essence de la communauté politique. Comment pourrait-on donc penser une communauté après l'époque de la disparition? Devrait-on dire alors que la disparition est la crise de la crise, l'impossibilité face à la possibilité même de l'exercice de la politique - du partage du sensible (Rancière) -, au sens simondonien d'une interruption du processus d'individuation psycho-sociale? Il faudra donc parler de la post-politique, comme Lyotard parle du post-humain, de l'inhumain? Il faudra donc inscrire le phénomène de la disparition dans le processus global (dit postmoderne) de l'absence de destination, c'est-à-dire, de l'impossibilité de l'inscription de l'événement? Nous allons essayer, dans notre recherche, de répondre à ces questions à partir de la détermination de ce qu'est une image politique propre à l'art latino-américain au cours des trois dernières décennies.

Il s'agira après l'époque de la disparition, d'une (anti)communauté post-politique de l'inhumain. Les catégories mobilisées par Lyotard, seront travaillées afin de penser ce qu'au début nous appelions le phénomène d'effondrement du sens. A partir des années 80 Lyotard a revitalisé la notion kantienne du sublime pour en dégager le sens d'une expérience face à ce que l'on a appelé l'irreprésentable (Nous nous occuperons aussi de la considération des repaires critiques de Rancière face à cette question). Dans son ouvrage philosophique, peut-être le plus dense, Le différend, Lyotard a mis en évidence l'impossibilité, après la Shoah, de toute communication, de toute destination linguistique d'une communauté. Il s'agirait donc là de l'impossibilité même d'une lutte quelconque pour l'espace politique, pour s'approprier une zone de sensibilité qui restait jusque là dans la société comme un vide obscur, et donc de l'impossibilité de l'exercice politique dans son sens classique, qui finalement est celui qui prédomine dans la philosophie politique de Rancière. Selon notre lecture, tant l'expérience - car, dans son sens kantien, il s'agit bien là d'une expérience - du sublime que celle du différend devront être considérées comme l'horizon ontologique propre à une « époque de la disparition ».

Ainsi, nous ne pourrons pas éviter de faire un rapport entre les notions de différend et de mésentente. La mésentente sera définie en tant qu'agir politique qui permet la crise de l'espace politique (le partage du sensible) par l'apparition d'une part (la part des sans part) qui n'existait pas avant de la répartition politique des espaces et des voix, des lieux et des discours. Le disparu est justement celui que ne peut plus apparaître, qui n'apparaîtra plus jamais, donc c'est celui pour qui est nié pour toujours la possibilité de partager la sensibilité politique commune ; un mort peut toutefois apparaître, il est là, histoire de l'enterrement comme essentiel à l'histoire de l'humanité elle-même, il est là, histoire du culte aux morts dans les lieux où demeure sa mémoire. Cependant, nous pourrons dire, en suivant Rancière que la " question » des disparus aujourd'hui est déjà advenue dans l'espace de la discussion juridico-politique, grâce aux actions des 
familiers (Madres de la Plaza de Mayo, par exemple), qui ont poussé l'espace politique jusqu'à le briser et le transformer en l'obligeant à y inclure le nom des « sans nom », la trace des sans trace, les disparus - tout comme autrefois les prolétaires, les femmes, etc. ont brisé l'espace politique pour être reconnus (eux, qui étaient justement, par définition, ceux que l'espace politique ne reconnaissait pas) en changeant, en même temps, la constitution elle-même de la communauté. Mais nous pourrons dire, en effet, par rapport aux familiers des disparus, qui ont construit un sens politique pour l'agir politique, et de cette façon-là ont pu être reconnus comme des acteurs (eux, en tant que familiers des disparus) politiques de nos sociétés contemporaines, que la question notre question, la question de notre recherche - est plutôt celle des disparus euxmêmes. C'est donc la question du comment les inscrire dans la communauté, en considérant que les morts ont toujours été inscrits - cf. les cimetières et leur histoire -, c'est la question du comment penser ce qui n'a pas été inscrit, comment penser un événement qu'on ne peut inscrire? Revient, nous le voyons, la question de l'inscription. A une époque où la communauté politique doit partager, pour se constituer, pour se (des)individuer, des faits politiques qui sont ininscriptibles - en en attaquant le fait peut-être fondateur de toute communauté, à savoir le fait de l'inscription de la loi sur les diverses supports sociaux, le corps notamment -, et en même temps doit (cette communauté toujours (im)possible) compter les fantômes en tant qu'acteurs politiques - c'est pour cela qu'une philosophie politique aujourd'hui doit être en même temps une philosophie des fantômes - ; il faudra parler du différend plutôt que de la mésentente.

Un autre enjeu fondamental de notre recherche va être posé par la question du rapport étroit entre la question de la disparition - c'est-à-dire la question de la réalité des sujets qui ont existé mais qui, une fois morts ont disparus, qu'on ne peut plus les inscrire, la question de la réalité (question ontologique donc) de ces véritables fantômes - et ce que Lyotard appelait les immatériaux, moment où l'inscription ne se fait plus sur des supports matériels, une " question pressante » selon Lyotard lui-même : « avec la perte du matériau, la destinée en chômage?» Quand on considère comment a été, particulièrement l'appareil photographique et sa capacité d'inscrire-écrire l'image sur un support matériel - en rendant possible l'expérience du ça a été barthésien - celui qui a permis aux familiers des disparus de prouver la réalité de l'existence de leurs enfants, et en même temps - par rapport à la reproductibilité de l'image photographique, reproductibilité aussi de leur vérité - peupler les villes, les rues et les lieux de la disparition de cette vérité-là, nous constatons comment nous avons dépassé l'époque de la matérialité des supports d'inscription vers l'époque de son immatérialité. Nous pouvons nous demander aussi quel est le statut - s'il y en a un - aujourd'hui d'une certaine preuve par l'image, et s'il existe cette possibilité même d'une vérité politique de l'image? Qu'est-ce que la politique de l'image après l'époque de la disparition? Comment inscrire, aujourd'hui, l'événement de la disparition ? Est-ce que la disparition est un événement? Il s'agira donc avant tout de la question de l'inscription de ce qui par définition ne peut plus être inscrit. Voilà l'importance de œuvres d'artistes comme les chiliens Carlos Altamirano et Eugenio Dittborn et les argentins Marcelo Brodski et Fernando Traverso, que nous allons commenter. 
INDEX

Mots-clés : Argentine, catastrophe, Chili, crime politique, Deleuze, désastre, disparition,

événement, fantasme, image, image politique, immatériaux, Lyotard, mémoire, photographie, Rancière, régimes totalitaires, ruine, Simondon, surface d'inscription, témoignage, trace 